\title{
Expresión de citoquinas Th17 y su correlación con periodontopatógenos y el área periodontal inflamada en pacientes con periodontitis crónica
}

\author{
Th17 cytokines expression and its correlation with \\ periodontopathogens and periodontal inflammed surface area \\ (PISA) in patients with chronic periodontitis
}

\author{
Herane $A^{1}$, Chaparro $A^{1}$, Quintero $A^{1}$, Sanz $A^{1}$, Hernández $\mathrm{M}^{2}$, Gaedechens $\mathrm{D}^{3}$, Carrión $\mathrm{F}^{4}$, Inostroza $\mathrm{C}^{2}$
}

\begin{abstract}
RESUMEN
Antecedentes: La periodontitis es una enfermedad inflamatoria infecciosa que involucra una respuesta inmune del hospedero y se caracteriza por destrucción del hueso alveolar, el objetivo del estudio es analizar la expresión de citoquinas Th17 y su correlación con periodontopatógenos y el área periodontal inflamada en pacientes con periodontitis crónica. Método: Se realizó un estudio descriptivo exploratorio en el que se reclutaron 23 pacientes con diagnóstico de periodontitis crónica y un grupo control de 10 individuos sano/gingivitis. A todos los sujetos se les realizó un examen periodontal completo. Además, se utilizó el método PISA (Periodontal Inflamed Surface Area) para cuantificar el tamaño de la herida periodontal. Se recolectaron muestras de FGC, plasma y placa bacteriana para su análisis mediante técnica de ELISA de IL-17 A, IL-6, IL-23 y IL-10 y PCR para la determinación de la presencia de: P. gingivalis, T. denticola, T. forsythensis, A. actinomycetemcomitans, F. nucleatum y P. intermedia. Los datos fueron analizados utilizando estadística descriptiva y la asociación entre variables se estimó a través de modelos de regresión logística. Resultados: Se observó una tendencia al aumento, no significativa, de los niveles de IL-17A, IL-6 y IL-23 a nivel de FGC en los sujetos con periodontitis crónica $(p=0.716,0.784,0.421$, respectivamente). Los pacientes con periodontitis crónica presentaron una disminución de la IL-10 ( $p=0.012)$ y los niveles de IL-17A se correlacionaron positivamente con el área periodontal inflamada $(p=0.004)$. A nivel de los patógenos periodontales, se observó una asociación entre la presencia de: $P$. gingivalis, T. denticola, $T$. forsythensis y los niveles de IL-6 plasmática ( $p=0.017,0.033,0.024$, respectivamente). Rev. Clin. Periodoncia Implantol. Rehabil. Oral Vol. 6(3); 109-113, 2013.
\end{abstract}

Palabras clave: Periodontitis crónica, PISA, Th17, periodontopatógenos.

\section{ABSTRACT}

Objective: Periodontitis is an infectious and inflammatory disease that involves a host immune response and is characterized by alveolar bone destruction. Aim: Analyze the expression of Th17 cytokines and their correlation with periodontopathogens and periodontal inflamed area in patients with chronic periodontitis. Method: A case control study was performed. At the time of delivery, 23 cases of patients with periodontal diagnosis were enrolled in the study and 10 controls with gingivitis. The diagnosis involved a complete periodontal examination with periodontal Florida probe. Also we used the PISA (Periodontal Inflamed Surface Area) index to classify the groups. Plasma and GCF samples were collected and studied for protein expression by ELISA assays for IL17A, II6, IL23 and IL10. Plaque was analyzed by PCR for the determination of the presence of: P. gingivalis, $T$. denticola, T. forsythensis, F. nucleatum, P. intermedia and A. actinomycetemcomitans. Data was analyzed using descriptive statistics and the association between variables was estimated through logistic regression models. Results: There is a trend of increased GCF levels of IL17A, IL6, and IL23 with no significance. However there was an association between gingivitis and IL10 plasma and GCF levels $(p=0.012)$. In relation to the periodontal wound size, a correlation was observed between the levels of IL6 and IL10 in GCF. Analysis of periodontal pathogens, showed an association between the presence of: $P$. gingivalis, T. denticola, $T$. forsythensis and plasma levels of IL-6 ( $p=0.017,0.033$ and 0.024 , respectively).

Rev. Clin. Periodoncia Implantol. Rehabil. Oral Vol. 6(3); 109-113, 2013.

Key words: Periodontitis, PISA, Th17, periodontopathogens.

\section{INTRODUCCIÓN}

La periodontitis es una enfermedad infecciosa que se caracteriza por presentar inflamación crónica, la cual resulta en daño tisular extenso de las estructuras de soporte del diente, siendo así, una de las causas mas significativas de perdida de piezas dentarias en adultos ${ }^{(1,2)}$.

El biofilm bacteriano adherido a la superficie dentaria en estrecha relación con los tejidos periodontales es uno de los factores etiológicos más relevantes de esta enfermedad( ${ }^{(3)}$. La incidencia y progresión de la enfermedad periodontal depende de la compleja interacción entre las bacterias periodontopatógenas y las células del sistema inmune del huésped ${ }^{(4,5,6)}$. Estas interacciones están mediadas por citoquinas y quimioquinas producidas tanto por el huésped como por células presentes en el sitio de la inflamación ${ }^{(1,2,7)}$. Moutsopoulus describió lesiones enfermas que fueron poblados por abundantes células Th17, esta infiltración se relaciona a la infección, la inflamación crónica y a la patología del tejido. Experimentos in vitro revelan que $P$. gingivalis favorece la estimulación de la producción de citoquinas Th17 y promueve la producción de IL-6 ${ }^{(8)}$.

1. Cirujano-Dentista. Especialista en Periodoncia. Profesor Asistente. Departamento de Periodoncia. Facultad de Odontología, Universidad de los Andes. Chile. 2. Bioquímico. Magíster en Ciencias Biológicas. Mención Microbiología. Centro de Biología y Regeneración Oral (CIBRO). Facultad de Odontología, Universidad de los Andes. Chile.

3. Cirujano-Dentista. Estudiante del postítulo de especialidad en Periodoncia e Implantología quirúrgica. Universidad de los Andes. Chile.

4. Bioquímico. Doctorado en Inmunología. Laboratorio de Inmunología. Facultad de Medicina. Universidad de los Andes. Chile. 
En 1976, Page y Schroeder describieron la lesión periodontal desde el punto de vista histopatológico en cuatro etapas: Inicial, temprana, establecida y avanzada. Las lesiones inicial y temprana se caracterizan por un infiltrado inflamatorio donde predominan los neutrófilos y los macrófagos, mientras que las lesiones establecida y avanzada se caracterizan por la presencia de linfocitos T, B y principalmente células plasmáticas $^{(9)}$. La gingivitis se ha considerado como una lesión estable donde predominan los linfocitos $\mathrm{T}$, mientras que la periodontitis se considera una lesión avanzada en la cual predominan los linfocitos B y las células plasmáticas ${ }^{(10)}$. Por tanto, el papel que desempeñan los linfocitos $T$ es de vital importancia en la patogenia de la enfermedad periodontal.

Los linfocitos $T$ pueden subdividirse en dos grandes subgrupos de acuerdo a la expresión de las moléculas CD4 y CD8; linfocitos Thelper (Th) y linfocitos T citotóxicos (Tc), respectivamente ${ }^{(11)}$. Tanto los linfocitos T CD4+ como los CD8+ pueden clasificarse según el perfil de citoquinas que producen. Para las células CD4+ se han descrito dos perfiles: Th1 que producen IL-2, INF- $\gamma$, TNF- $\alpha$, TNF- $\beta$ y promueven respuestas mediadas por células esenciales para la eliminación de patógenos intracelulares. Las células Th2 median la inmunidad humoral y producen principalmente IL-4 e IL-5, las cuales inducen el crecimiento y diferenciación de células $B$ y células plasmáticas. Es de suponer que un balance entre las citoquinas Th1 y Th2 sea necesario para producir respuestas inmunes efectivas con el fin de controlar el agente agresor, en este caso la placa bacteriana. Las citoquinas presentes en el tejido periodontal enfermo están determinadas por la respuesta linfocitaria y son fundamentales en determinar que la enfermedad periodontal progrese o permanezca estable $^{(12)}$. Estudios recientes demuestran que el modelo clásico de las células T CD4 efectoras Th-1/Th-2 debe ser reinterpretado, dándole espacio a las células Th-17 dentro de las patogenias inflamatorias articulares como las enfermedades autoinmune. Se ha definido que los linfocitos Th17 caracterizados como un subtipo productor principal de IL-17, es antagonizado por los productos de los linajes Th1 y Th2 ${ }^{(13)}$. En humanos la IL-17 tiene una función proinflamatoria. Los linfocitos T reguladores Tregs, son una subpoblación especializada de linfocitos $T$ que actúa suprimiendo la activación del sistema inmunitario, manteniendo así la homeostasis de este sistema y favoreciendo la tolerancia hacia autoantígenos. La función anti-inflamatoria de Tregs supresora se caracteriza por la secreción de TGF-beta 1 e IL-10 ${ }^{(14)}$. Diversos hallazgos han involucrado a las células Th17 en lesiones crónicas de los tejidos periodontales y por lo tanto estarían implicadas en la reabsorción ósea del hueso alveolar de pacientes con periodontitis crónica severa. Los resultados de Okui y cols. $2012^{(15)}$, sugieren que en lesiones periodontales distintas citoquinas inflamatorias podrían gatillar la conversión de células Tregs (FOXP3+) en células TH17 productoras de IL-17. El balance entre células Th17 y Treg es crucial en la homeostasis del sistema inmune.

El objetivo general de este estudio es evaluar en fluido gingival crevicular (FGC) y plasma los niveles de expresión de citoquinas Th17 (IL17A e IL-23), citoquina proinflamatoria (IL-6) y una citoquina que contribuye a la actividad supresora Tregs (IL-10) y correlacionar su expresión con periodontopatógenos y el área periodontal inflamada (PISA) en pacientes con periodontitis y en un grupo control de individuos sanos/gingivitis.

\section{MATERIAL Y MÉTODO}

Se realizó un estudio descriptivo exploratorio de 33 individuos que acudieron a la unidad de diagnóstico del Centro Odontológico de Especialidades Médicas de la Universidad de los Andes (CESA), en el Hospital Parroquial de San Bernardo (HPSB), Región Metropolitana, Santiago, Chile. Todos los pacientes ingresaron voluntariamente al estudio y firmaron previamente un consentimiento informado. El estudio contó con la aprobación del comité de ética de la Facultad de Odontología y del Comité de Ética de la Universidad de los Andes.

A todos los pacientes se les realizó exámen periodontal completo en sitios molares, en el que se determinó profundidad al sondaje (PS), nivel de inserción clínica (NIC) e Índice de sangrado (IS). El examen fue realizado por un especialista en periodoncia previamente calibrado. Los pacientes fueron examinadas en los sillones dentales utilizando luz artificial y un instrumental de examen básico. (Espejo, pinza y sonda manual Periodontal Hu-Friedy Carolina del Norte). Además de realizar el examen periodontal se recopilaron datos personales, tales como; edad, estado civil, nivel educacional junto a antecedentes mórbidos, gineco-obstétricos y odontológicos.
Criterios de Inclusión

Se incluyeron en el estudio hombres y mujeres de entre 20-65 años de edad, que presenten al menos 14 dientes naturales en boca (excluyendo terceros molares).

\section{Criterios de Exclusión}

Haber recibido tratamiento periodontal en el último año, terapia antibiótica 0 antinflamatorios esteroidales o no esteroidales en los últimos 6 meses, Fumadores, embarazadas o en período de lactancia, enfermedades sistémicas relevantes que afecten el curso o severidad de la periodontitis.

\section{Determinación del Diagnóstico Periodontal}

Sano/Gingivitis: $\leq 10 \%$ de los sitios con $P S \geq 3 \mathrm{~mm}$ y un índice de sangrado $\leq 10 \%$. Sin pérdida de inserción clínica ${ }^{(16)}$.

Periodontitis: $>10 \%$ de los sitios con PS $\geq 3 \mathrm{~mm}$ y un índice de sangrado $>10 \%$. Con pérdida de inserción.

Determinación Tamaño Herida Periodontal Mediante el Método Pisa EI método PISA refleja el área de superficie de sangrado del epitelio del saco periodontal y a su vez el área de superficie de sangrado del epitelio del saco periodontal cuantifica la cantidad de tejido periodontal inflamado(17).

Se ha construido una hoja en Microsoft Excel para facilitar su cálculo. Está disponible en forma gratuita en la página web www. parsprototo. info. Se requiere rellenar con los datos de NIC, Recesión gingival e IS en 6 sitios por diente. También se puede utilizar las mediciones de PS.

Toma de Muestras de FGC para Inmunoensayo ELISA y para Detección de Periodontopatógenos por PCR

Se seleccionaron 4 sitios por cada cuadrante de mayor profundidad al sondaje. Luego de aislar el diente con tórulas de algodón, se removió la placa supra gingival cuidadosamente con una cureta $(\mathrm{Hu}-$ Friedy, Chicago, USA) sin tocar el margen gingival. Posteriormente se secó el sitio crevicular con aire de la jeringa triple. EI FGC se recolectó con 4 conos de papel endodóntico estériles número 40 (Dentsply Maillefer, Tulsa, OK, USA). Las puntas de papel se introdujeron en el surco/saco periodontal hasta sentir una mediana resistencia. Se dejaron en el sitio por 30 segundos. Se excluyeron los conos contaminados con saliva o sangre. Finalmente los conos se almacenaron en tubos Eppendorf (Biologix, Lenexa, $\mathrm{KS}$, USA) en frío a $-80^{\circ} \mathrm{C}$ para su posterior análisis.

\section{Toma de Muestras de Sangre Venosa Periférica}

Se tomaron $5 \mathrm{ml}$ de sangre venosa del antebrazo. Las muestras fueron depositadas en tubos vacutainer con anticoagulante EDTA (BD Vacutainer, Franklin Lakes, NJ, USA) y fueron centrifugadas a $3.000 \mathrm{rpm}$ durante 10 minutos (Spectrafuge 6C, Labnet ${ }$, Edison Township, NJ, USA). Luego se removió el sobrenadante (plasma) el que se almacenó en tubos Eppendorf rotulados a $-80^{\circ} \mathrm{C}$ para su posterior análisis. La toma de muestras y su procesamiento fueron realizados por una enfermera calificada.

\section{Extracción de DNA Genómico}

Se utilizaron dos conos de cada muestra, los cuales se colocaron en tubos Eppendorf estériles rotulado que contenían $200 \mu \mathrm{L}$ de agua libre de nucleasas (IDt $\circledast$, San Diego, CA, USA). Se incubaron las muestras durante 10 minutos a una temperatura de $94^{\circ} \mathrm{C}$ en termoblock (BioProducts $\AA$, West Palm Beach, FL, USA) y posteriormente durante 10 minutos una temperatura de $-20^{\circ} \mathrm{C}$. Finalmente se centrifugó durante 5 minutos.

Una vez realizada la extracción de $A D N$ se retiró el sobrenadante de producto denaturado $(120 \mu \mathrm{L})$, se introdujo a un tubo Eppendorf (Biologix, Lenexa, KS, USA) estéril rotulado y se almacenó a $-20^{\circ} \mathrm{C}$. Se desecharon los conos de la muestra.

\section{Reacción en Cadena de la Polimerasa (PCR)}

Se utilizó la técnica molecular PCR convencional para determinar la presencia o ausencia de patógenos periodontales en la placa subgingival. Las bacterias analizadas fueron Porphyromonas 
gingivalis $(P g)$, Tannerella forsythia $(T f)$, Treponema denticola $(T d)$, Prevotella intermedia (Pi), Fusobacterium nucleatum (Fn) y Agregatibacter actinomycetemcomitans $(A a)$. Los partidores de PCR fueron mandados a hacer a IDt@ (San Diego, CA, USA) y fueron utilizados en la determinación de la presencia o ausencia de las especies bacterianas (Tabla 1).

Posteriormente, se llevaron los tubos de PCR a termociclador multigene TM (Labnet $\circledast$, Edison Township, NJ, USA). El programa de PCR fue el mismo para todas las muestras y se realizó con un ciclo de denaturación por 5 minutos a $94^{\circ} \mathrm{C}$ y 40 ciclos de denaturación a $94^{\circ} \mathrm{C}$ por 30 segundos, apareamiento a $55^{\circ} \mathrm{C}$ por 30 segundos y elongación a $72^{\circ} \mathrm{C}$ por 45 segundos. La elongación final fue a $72^{\circ} \mathrm{C}$ por 10 minutos y un ciclo final a $15^{\circ} \mathrm{C}$ por 59 minutos.

Los fragmentos de DNA productos de la reacción de PCR fueron visualizados en geles de agarosa (Seakem ${ }^{\circledR}$ LE, Lonza, Allendale, NJ, USA) al $2 \%$ con Buffer TBE 1X (Tris, Borato, EDTA) (Promega(TM), Madison, WI, USA) y SybrSafe (Life Technologies Corporation, Carlsbad, CA, USA) y SybrSafe (Invitrogen Tm). Las imágenes de los geles fueron adquiridas en un sistema de foto documentación Enduro tm GDS (Labnet ${ }^{\circledR}$, Edison Township, NJ, USA).

Determinación de los Niveles de IL-17, IL-23, IL-6 y IL-10 en Muestras de FGC y Plasma

Se utilizó la técnica de inmunoensayo ELISA (acrónimo del inglés Enzyme-Linked Immuno Sorbent Assay). En cada una de las muestras, a partir del mismo volumen de FGC y de plasma de pacientes y controles se realizó el ensayo por inmunoabsorción ligado a enzimas. Se determinó los niveles de IL-17A, IL-23, IL-6 y IL-10. Se utilizaron los kits Human IL-17A ELISA Ready Set Go! $§$, Human IL-23 ELISA Ready Set Go!@, Human IL-6 ELISA Ready Set Go!@ y Human IL-10 ELISA Ready

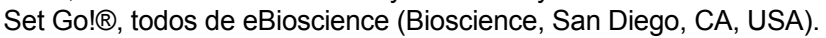

\section{Análisis Estadístico}

Los resultados de las muestras obtenidas no presentaron una distribución normal por lo tanto se realizaron test no parámetricos para analizar los datos. El análisis estadístico se hizo mediante el software Systat versión 13. Se utilizó el test de Mann Whitney para analizar las variables continuas cuantitativas. Se consideró un valor $p<0,05$ para determinar diferencias significativas entre grupos y con un intervalo de confianza del $95 \%$. Además, se utilizó el test de Spearman para determinar la correlación entre las variables. Los resultados obtenidos se muestran en Tablas y Figuras.

Tabla 1. Partidores usados en el análisis con PCR convencional.

\begin{tabular}{|c|c|c|c|}
\hline Bacteria & $\begin{array}{l}\text { Nombre } \\
\text { Partidor }\end{array}$ & Secuencia 5'-3' & $\underset{\text { pb }}{\text { Tamaño }}$ \\
\hline \multirow{2}{*}{$\begin{array}{l}\text { T. denticola } \\
\text { (T.d.) }\end{array}$} & Tden-1 & taa tac cgs atg tgc tca ttt aca $t$ & \multirow[t]{2}{*}{316} \\
\hline & Tden-2 & tac aag aag cat tcc ctc ttc ttc tta & \\
\hline \multirow{2}{*}{$\begin{array}{l}\text { T. forsythia } \\
\text { (T.f.) }\end{array}$} & Tfor-1 & tac agg gga ata aaa tga gat acg & \multirow[t]{2}{*}{745} \\
\hline & Tfor-2 & acg tca tcc cca cct tcc tc & \\
\hline \multirow{2}{*}{$\begin{array}{c}\text { P. gingivalis } \\
\text { P.g.) }\end{array}$} & Pgin-1 & agg cag ctt gcc ata ctg cg & \multirow[t]{2}{*}{404} \\
\hline & Pgin-2 & act gtt agc aac tac cga tgt & \\
\hline \multirow{2}{*}{$\begin{array}{l}\text { A. actinomycetemcomitans } \\
\text { (A.a.) }\end{array}$} & Aa-1 & acg tca tcc cca cct tcc tc & \multirow[t]{2}{*}{360} \\
\hline & Aa-2 & acg tca tcc cca cct tcc tc & \\
\hline \multirow{2}{*}{$\begin{array}{l}\text { F. nucleatum } \\
\text { (F.n.) }\end{array}$} & Fspp-1 & gga ttt att ggg cgt aaa gc & \multirow[t]{2}{*}{167} \\
\hline & Fspp-2 & ggc ttt cct aca aat atc tac gaa & \\
\hline \multirow{2}{*}{$\begin{array}{l}\text { P. intermedia } \\
\text { (P.i.) }\end{array}$} & Pint-1 & ttt gtt ggg gag taa agc ggg & \multirow[t]{2}{*}{650} \\
\hline & Pint-2 & tca aca tct ctg tat cct gcg t & \\
\hline
\end{tabular}

Secuencia de partidores para los genes específicos de bacterias T.d., T.f., P.g., A.a., F.n. y P.i.
Tabla 2. Características clínicas en grupos Control (C) y Periodontitis crónica (PC).

\begin{tabular}{|l|c|c|c|c|}
\hline $\begin{array}{l}\text { Característica } \\
\text { clínica }\end{array}$ & Diagnóstico & $\mathbf{N}$ & $\begin{array}{c}\text { Promedio } \\
\mathbf{\pm} \mathrm{DS}\end{array}$ & valor P \\
\hline Edad (años) & $\mathrm{C}$ & 10 & $27.80 \pm 4.52$ & 0.088 \\
\cline { 2 - 4 } & PC & 23 & $36.17 \pm 10.97$ & \\
\hline \multirow{2}{*}{ IS (\%) } & $\mathrm{C}$ & 10 & $2.88 \pm 0.95$ & \multirow{2}{*}{$0.0001^{\text {***}}$} \\
\cline { 2 - 4 } & PC & 23 & $70.15 \pm 19.25$ & \\
\hline Área $\left(\mathbf{c m}^{2}\right)$ & C & 10 & $0.83 \pm 0.46$ & \multirow{2}{*}{$0.0001^{\text {***}}$} \\
\cline { 2 - 4 } & PC & 23 & $13.68 \pm 7.55$ & \\
\hline
\end{tabular}

Las características clínicas que se evaluaron fueron: Edad en años, índice de sangrado (IS) mediante sondaje periodontal y área de la herida periodonta $\left(\mathrm{cm}^{2}\right)$ con método PISA del grupo control (C) y del grupo con periodontitis crónica (PC). Todos los valores se expresan como promedio \pm Desviación estándar (DS). ${ }^{* * *} \mathrm{p}<0.001$.

Tabla 3. Niveles de expresión de citoquinas $(\mathrm{pg} / \mathrm{ml})$ en grupos Control (C) y Periodontitis crónica (PC) en Plasma y FGC.

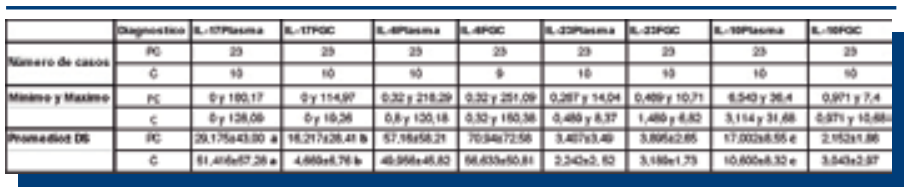

En el grupo control (C) y en el grupo con periodontitis (PC) se evaluaron los niveles de expresión de citoquinas en plasma y FGC, mediante Test de Elisa específicos para IL-17 A, IL-6, IL-23 e IL-10. Los valores se expresan como promedios en $\mathrm{pg} / \mathrm{ml} \pm$ Desviación Standard (DS). ${ }^{\mathrm{a}} \mathrm{p}=0.312,{ }^{\mathrm{b}} \mathrm{p}=0.716$ $y^{c} p=0.012$.

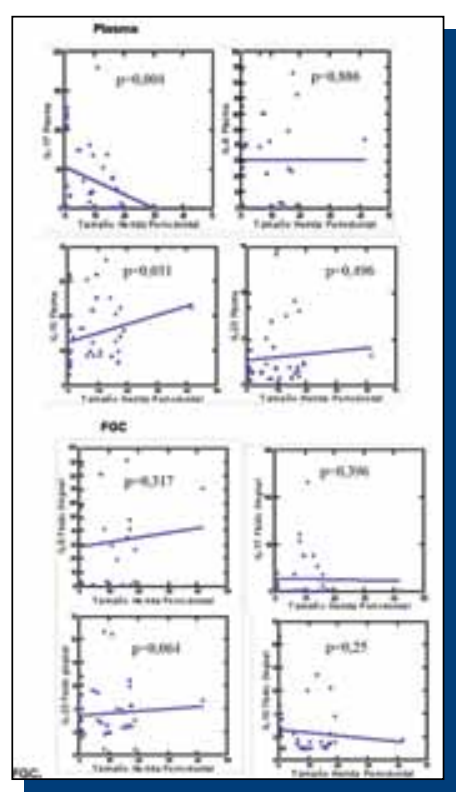

Se describe la correlación entre el tamaño de la herida periodontal y los niveles plasmáticos y de FGC de citoquinas IL-17, IL-23, IL-10 e IL-6 evaluadas mediante test de ELISA. Se muestran los valores $p, R$ y $R^{2}$ correspondientes a cada gráfico de la figura. ${ }^{* *} p<0.01$.

Figura 1. Correlación entre el área de la herida periodontal y niveles de citoquinas (pg/ml) IL-17A, IL-6, IL-23 y IL-10 en plasma y FGC. 


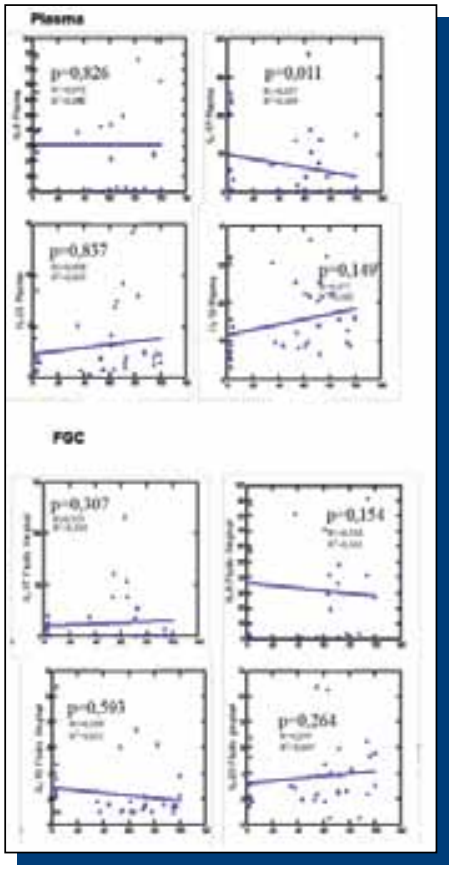

Se describe la correlación entre el \% de IS y los niveles plasmáticos y de FGC de citoquinas IL-17A, IL-23, IL-10 e IL-6 evaluadas mediante test de ELISA. Se muestran los valores $p, R$ y $\mathbf{R}^{2}$ correspondientes a cada grafico de la figura.

Figura 2. Correlación entre \% de Índice de sangrado y niveles de citoquinas (pg/ml) IL-17A, IL-6, IL-23 y IL-10 en plasma y FGC.

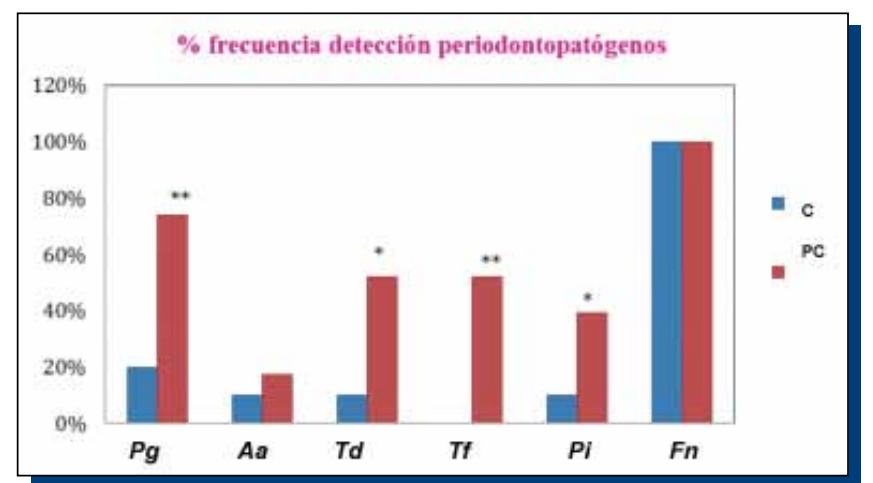

Comparación de los \% de frecuencia de periodontopatogenos en grupos Control (C) y Periodontitis crónica $(\mathrm{Pc})$. Porphyromona gingivalis $(P g)$ $(p=0.004)$, Agregatibacter actinomytemcomitans $(A a)(p=0.586)$, Treponema denticola $(T d)(p=0.0239), T$. forsythensis $(T f)(p=0.004)$, Prevotella intermedia $(P i)(p=0.094)$, Fusobacterium nucleatum $(F n)(p=0.024) .{ }^{*} p<0.05,{ }^{* *} p<0.01$.

Figura 3. Porcentaje de frecuencia de detección de periodontopatógenos en ambos grupos de estudio.

\section{RESULTADOS}

\section{Características Clínicas}

En el presente estudio se analizaron un total de 33 sujetos. El grupo de pacientes enfermos con periodontitis crónica $(\mathrm{Pc})$ incluyó 23 sujetos, dentro de ellos 9 hombres y 14 mujeres, con un rango de edad 20-57 años (36.174 +/ 10.965) y en el grupo control, sujetos sanos/ gingivitis $(C)$ incluyó 10 sujetos de ellos 4 hombres y 6 mujeres con un rango de edad de $24-38$ años $(27.8+/-4.517)$. Las características clínicas Índice de sangrado (IS) y área de la herida periodontal de los sujetos incluidos en este estudio se muestran en la Tabla 2. En los pacientes con periodontitis crónica se observó un aumento significativo del IS $(p=0.0001)$ y un mayor área de la herida periodontal $(p=0.0001)$ en comparación a los sujetos controles. Adicionalmente, se determinó una asociación entre el área de la herida periodontal $\left(\mathrm{cm}^{2}\right)$ y el diagnóstico de la patología periodontal $(p=0.001)$.
Análisis de los Niveles de Citoquinas Th17 (IL17A, IL23), IL6 e IL10 en Muestras de Plasma Sanguíneo y FGC

Los valores promedios, mínimo y máximo y las desviaciones estándar de cada citoquina están expresados en la Tabla 3. Se compararon los niveles de citoquinas entre ambos grupos. Se observaron diferencias significativas en los niveles de IL-10 plasmática entre grupo $P C$ y controles $(p=0.012)$. No se encontraron diferencias significativas entre los grupos en los niveles de IL-17A, IL-6 y IL-23 en plasma como en FGC y en los niveles de IL-10 a nivel de FGC.

Se observó una correlación positiva entre el área de herida periodontal e índice de sangrado (IS) $(p=0.0001)$. También se muestra una correlación positiva entre el área de herida periodontal con IL-17A e IL-10 plasmática, observándose que al disminuir el área disminuyen los niveles de IL-17 plasmática $(\mathrm{p}=0.004)$ y que al aumentar IL-10 plasmática aumenta el área $(p=0.031$ ) (Figura 1).

\section{Detección de Periodontopatógenos según Grupo de Estudio}

Al correlacionar la presencia de microorganismos con el diagnóstico periodontal (Figura 4) se observó una asociación entre periodontitis crónica y la presencia de Porphyromona gingivalis $(p=0.004)$, Treponema denticola $(p=0.0239), T$. forsythensis $(p=0.004)$ y Fusobacterium nucleatum ( $p=0.024)$.

En relación al análisis de las citoquinas y los microorganismos en los pacientes con periodontitis crónica, se observó una correlación entre los niveles de IL-6 plasmática con la presencia de Porphyromona gingivalis $(p=0.017)$, Treponema denticola $(p=0.033)$ y $T$. forsythensis $(p=0.024)$.

\section{DISCUSIÓN}

En nuestro estudio abordamos el papel de la respuesta $\mathrm{TH} 17$ inducida por periodontopatógenos y lo correlacionamos con el tamaño de la herida periodontal en pacientes con periodontitis crónica. El análisis de las características clínicas del grupo de estudio muestra que de los 33 sujetos reclutados, el porcentaje de Índice de Sangrado y el área del tamaño de la herida periodontal de 23 pacientes con periodontitis crónica (PC) aumenta significativamente en comparación a los 10 individuos del grupo control con gingivitis $(C)$. Determinamos una asociación significativa entre PC y la presencia de Porphyromona gingivalis, Treponema denticola y $T$. forsythensis. En la enfermedad periodontal la presencia de citoquinas proinflmatorias como IL-6 está ampliamente documentada, Reinhardt y cols., encontraron niveles significativamente mayores de IL-6 en FGC de pacientes con periodontitis refractaria en comparación con sujetos estables $^{(15)}$. Lee y cols., observaron niveles significativamente mayores de IL-6 en FGC en sitios activos en comparación con sitios inactivos ${ }^{(16)}$. Nuestros hallazgos muestran una tendencia al aumento de IL-6 a nivel de FGC y plasma. Estos resultados pueden significar que los niveles de este mediador inflamatorio en pacientes periodontales puede verse influenciado por diversos factores, entre ellos la composición bacteriana local. Tamai y col., observaron que infecciones mixtas con $P$. gingivalis, con $T$. forsythia o $T$. denticola actúan sinérgicamente aumentando la producción de IL-6 ${ }^{(18)}$. En el presente estudio se observa una correlación positiva entre la presencia de los tres periodontopatógenos del grupo rojo ( $P$. gingivalis, $T$. denticola y $T$. forsythensis) y el diagnóstico periodontal. Geivelis y cols., observaron una correlación positiva entre profundidad al sondaje e IL-6 en FGC(17).

El rol de IL-17A en la enfermedad periodontal aun es controversial. Observamos una tendencia al aumento de IL-17A a nivel de FGC en el grupo PC, pero no observamos diferencias significativas en los niveles de IL-23 a nivel de FGC o plasma entre los dos grupos. La tendencia al aumento de los niveles de IL17A en FGC podría ser consecuencia del desarrollo de la respuesta inmune a nivel local por la pérdida ósea asociada al grupo PC. A nivel plasmático no se observaron diferencias en IL-17A entre los dos grupos de estudio. En relación a IL-23 en el grupo PC hay una tendencia al aumento en los niveles de IL-23 en FGC y plasma. La expresión de IL-17A en el grupo PC no fue detectada en 9 muestras de plasma y 13 de FGC y a la vez en 3 muestras de plasma y 5 de FGC en el grupo control. La no detección de IL-17A en varias de las muestras de FGC y plasma de PC junto a la dispersión de los datos y un pequeño tamaño muestral podrían ser factores claves en explicar por qué no obtuvimos diferencias significativas entre ambos grupos. 
Con respecto a la expresión de una citoquina supresora como la IL-10, nuestros resultados muestran una mayor expresión de IL-10 en muestras de plasma en pacientes con PC en relación a controles, sin embargo, a nivel de FGC los niveles de IL-10 muestran una tendencia al aumento en el grupo control.

Otros estudios ${ }^{(8)}$ explican que esta promoción de las respuesta Th17 es promocionada por proteasas especificas secretadas por $P$. gingivalis, que degradarían diferencialmente a citoquinas fundamentales. A este respecto, la IL-12 era muy degradado por $P$. gingivalis, mientras que la IL-1 $\beta$ fue más resistente a la proteólisis.

Nuestros datos confirman que bacterias como $P$. gingivalis podrían orquestar la inflamación en periodontitis crónica.

Actualmente, se utilizan una gran variedad de modelos para la definición de casos de patología periodontal ${ }^{(20,21,22)}$. Debido a esto Nesse y col., desarrollaron un método, que cuantifica el área de tejido periodontal inflamado. Este método se conoce como PISA (Periodontal Inflamed Surface Area) $)^{(13)}$. En nuestro estudio observamos una correlación positiva significativa entre el tamaño de la herida y el IS es decir, que al aumentar el área de la herida periodontal aumenta el IS. Además, detectamos una correlación positiva significativa entre el área de la herida periodontal y los niveles de IL-17A plasmática. De lo anterior podemos inferir que el tamaño de la herida periodontal se correlaciona con los niveles de mediadores proinflamatorios presentes. Proponemos al método PISA como un parámetro validado para cuantificar el estado inflamatorio del tejido periodontal enfermo.

La complejidad de la respuesta inmune inflamatoria en las enfermedades periodontales ha sido ampliamente estudiada, proponiéndose múltiples hipótesis al respecto. Un eje novedoso es el del balance entre la autoinmunidad y tolerancia, representada por las respuestas celulares tipo Th17 y $\mathrm{T}$ reguladora (Tregs), respectivamente. Sin embargo, se necesitan más estudios para abordar si existe una conversión Treg-Th17 durante las respuestas inflamatorias contra periodontopatogenos. Para contribuir al entendimiento con investigaciones de este tipo y aportar con alguna aplicación práctica, útil para el diagnóstico y/o tratamiento de diferentes patologías orales ${ }^{(23)}$.

\section{CONFLICTO DE INTERESES} ningún tipo.
Los autores declaran no presentar conflictos de interés de

\section{REFERENCIAS BIBLIOGRÁFICAS}

1. Genco RJ, Grossi SG, Ho A, Nishimura F, Murayama Y. A proposed model linking inflammation to obesity, diabetes, and periodontal infections. J Periodontol, 2005; 76: 2075-2084.

2. Ghilardi N, Kljavin N, Chen Q, Lucas S, Gurney A, de Sauvage FJ. Compromised humoral and delayed-type hypersensitivity responses in IL-23-deficient mice. $J$ Immunol, 2004; 172: 2827-2833.

3. Kubar A, Saygun I, Ozdemir A, Yapar M, Slots J. Real-time polymerase chain reaction quantification of human cytomegalovirus and Epstein-Barr virus in periodontal pockets and the adjacent gingiva of periodontitis lesions. J Periodontal Res, 2005; 40: 97-104.

4. Page RC. Gingivitis. J Clin Periodontol, 1986; 13: 345-355.

5. Payne WA, Page RC, Ogilvie AL, All WB. Histopathological features of the inicial and early stages of experimental gingivitis in man. J Periodontal Res, 1975; 10: 51-64. 6. Schroeder HE, Múnzel-Pedrazzoli S, Page RC. Correlated morphometric and biochemical analysis of early chronic gingivitis in man. Arch Oral Biol, 1973; 18: 899-923. 7. Pradee AR, Hadge P, Chowdhry S, Patel S, Happy D. Exploring the role of Th1 cytokines: Interleukin-17 and interleukin-18 in periodontal health and disease. J Oral Sci, 2009; 51(2): 261-266.

8. Belardelli F, Ferrantini M. Cytokines as a link between innate and adaptive antitumor immunity. Trends Immunol, 2002; 23: 201-208.

9. Seymour GJ, Taylor JJ. Shorts and whispers: An introduction to immunoregulation in periodontal disease. Periodontol 2000, 2004; 35: 9-13

10. Savak T, Chabaud M, Moossee P, Natwig JB. IL-17 is produced by some pro inflammatory Th1 cell/Th0 cells but only by Th2 cells. J Immunol, 1999; 162: 1246-1251. 11. Weaver CT, Hatton RD, Mangan PR, Hamington LE. IL-17 family cytokines and the expanding diversity of effector T cell lineages. Annu Rev Immunol, 2007; 25: 821-852. 12. Weaver CT, Harrington LE, Mangan PR, Gavrieli M, Murphy KM. Th17: An effector CD4 T cell lineage with regulatory T cells. Immunity, 2006; 24: 677-688.

13. Nesse W, Abbas F, van der Ploeg I, Spijkervet FKL, Dijkstra PU, Vissink A. Periodontal inflamed surface area: Quantifying inflammatory burden. J Clin Periodontol, 2008; 35: 668-673.
14. Yu JJ, Ruddy MJ, Wong GC. An essential role for IL-17 in preventing pathogeninitiated bone destruction: Recruitment of neutrophils to inflamed bone requires IL-17 receptor-dependent signals. Blood, 2007; 109: 3794-3802.

15. Reinhardt RA, Masada MP, KaldahI WB et al. Gingival fluid IL-1 and IL-6 levels in refractory periodontitis. J Clin Periodontol, 1993; 20: 225-231.

16. American Academy of Periodontology. International Workshop for a Classification of Periodontal Diseases and Conditions. Papers. Oak Brook, Illinois, October 30-November 2, 1999. Ann Periodontol, 1999; 4: 1-112.

17. Geivelis M, Turner CW, Pederson ED, Lamberts BL. Measurements of IL-6 in gingival crevicular fluid from patients with destructive periodontal disease. $J$ Periodontol, 1993; 64: 980-983.

18. Tamai R, Deng X, Kiyoura Y. Porphyromonas gingivalis with either Tannerella forsythia or Treponema denticola induces synergic IL- 6 production by murine macrophage-like J774.1 cells. Anaerobe, 2009 Jun; 15(3): 87-90.

19. Takahashi K, Azuma T, Motohira H, Kinane DF, Kitetsu S. The potential role of interleukin-17 in the immunopathology of periodontal disease. J Clin Periodontol, 2005; 32: 369-374.

20. Scannapieco FA, Bush RB, Paju S. Associations between periodontal disease and risk for atherosclerosis, cardiovascular disease, and stroke. A systematic review. Ann Periodontol, 2003a; 8: 38-53.

21. Khader YS, Taani Q. Periodontal diseases and the risk of preterm birth and low birth weight: a meta-analysis. J Periodontol, 2005; 6, 161-165.

22. Vettore MV, Leal MD, Leao AT, da Silva AM, Lamarca GA, Sheiham A. The relationship between periodontitis and preterm low birthweight. J Den Res, 2008 87: 73-78.

23. Carré L, Franco ME, Henríquez L, García-Sesnich J, Dutzan N, Aguillón JC, Gamonal J. Presence of T regulatory cells in chronic periodontitis. Rev Clin Periodoncia Implantol Rehabil Oral, 2010; 3(3): 128-131. 